\title{
Analytical Study of Sandwich Structures using Euler-Bernoulli Beam Equation
}

\author{
Hui Xue ${ }^{1}$, H. Khawaja ${ }^{2, \text { a) }}$ \\ ${ }^{1}$ Master Student, Department of Computer Science and Computational Engineering, UiT The Arctic University \\ of Norway, Narvik, Norway \\ ${ }^{2}$ Associate Professor, Department of Engineering and Safety, UiT The Arctic University of Norway, Troms $\phi$, \\ Norway \\ a) Corresponding author: hassan.a.khawaja@uit.no
}

\begin{abstract}
This paper presents an analytical study of sandwich structures. In this study, the Euler-Bernoulli beam equation is solved analytically for a four-point bending problem. Appropriate initial and boundary conditions are specified to enclose the problem. In addition, the balance coefficient is calculated and the Rule of Mixtures is applied. The focus of this study is to determine the effective material properties and geometric features such as the moment of inertia of a sandwich beam. The effective parameters help in the development of a generic analytical correlation for complex sandwich structures from the perspective of four-point bending calculations. The main outcomes of these analytical calculations are the lateral displacements and longitudinal stresses for each particular material in the sandwich structure.
\end{abstract}

\section{INTRODUCTION}

The Euler-Bernoulli beam theory states that stresses vary linearly with the distance from the neutral axis [1, 2]. The classic formula for determining the longitudinal stress in a beam, as shown in Fig. 1 under simple bending, is given in Equation (1):

$$
\sigma_{x}=\frac{M|c|}{I}
$$

where $\sigma_{x}$ is the longitudinal stress in $\mathrm{Pa}, \mathrm{M}$ is the moment about the neutral axis in $\mathrm{Nm}, \mathrm{C}$ is the perpendicular distance from the neutral axis in $m$ and $I$ is the second moment of area about the neutral axis in $m^{4}$.

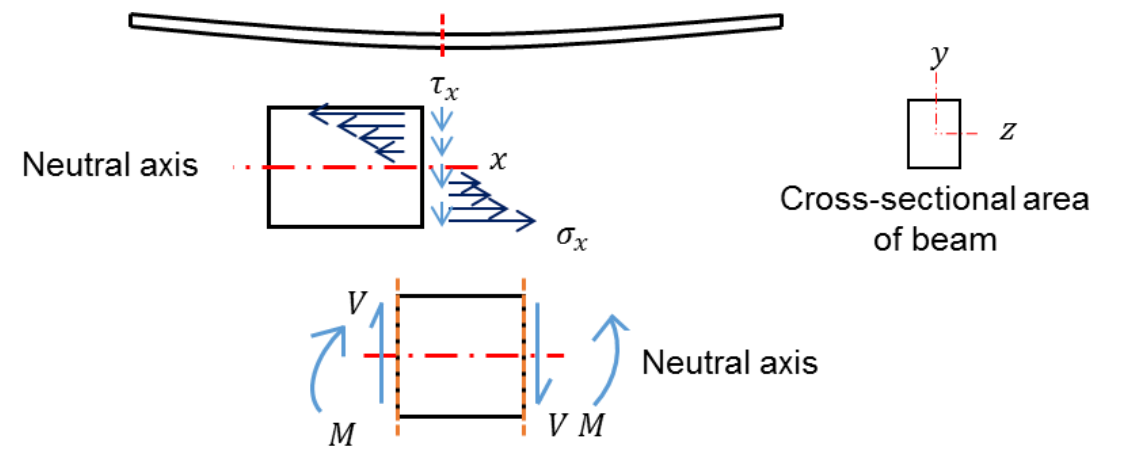

FIGURE 1. Longitudinal stress $\left(\sigma_{x}\right)$, shear stress $\left(\tau_{x}\right)$, shear force $(V)$ and bending moment $(M)$ in a beam

Deflection in the beam is shown in Fig. 2 In a bending beam, the strain $\varepsilon$ can be expressed by the radius of the neutral axis and the distance of the surface from the neutral axis. A correlation can be written as shown in Equation (2): 

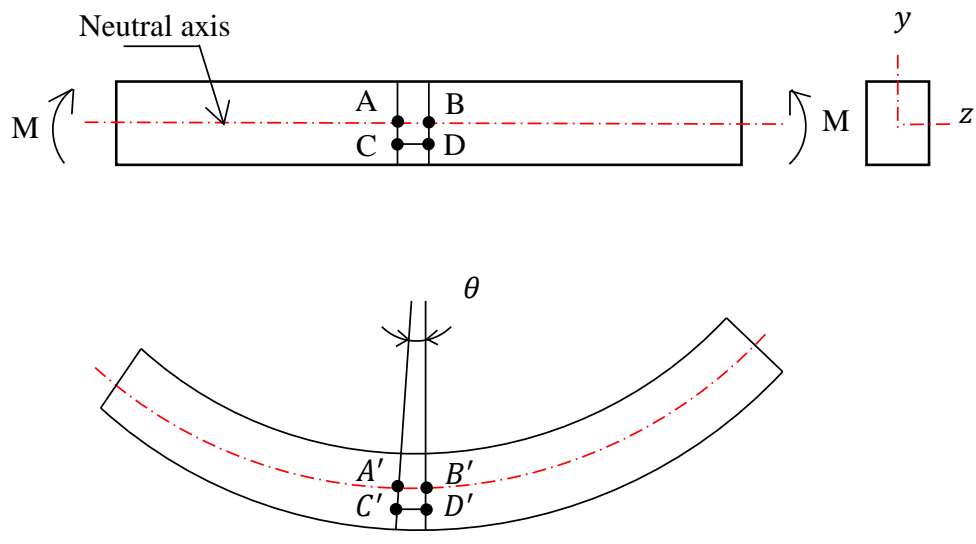

FIGURE 2. Longitudinal strain $\left(\varepsilon_{x}\right)$ in a bending beam

$$
\frac{C^{\prime} D^{\prime}}{A^{\prime} B^{\prime}}=\frac{(R+c) \theta}{R \theta}=\frac{R+c}{R}
$$

where $R$ is the radius of the neutral axis, $c$ is the distance from the neutral axis and $\theta$ is the slope in radians.

Thereafter, the strain $\varepsilon_{x}$ at layer at $C^{\prime} D^{\prime}$ is shown in Equation (3), where the line $C D$ is in the original layer so that the length $C D=A B$.

$$
\varepsilon_{x}=\frac{C^{\prime} D^{\prime}-C D}{C D}=\frac{C^{\prime} D^{\prime}-C D}{A B}=\frac{C^{\prime} D^{\prime}}{A B}-1
$$

Since $A^{\prime} B^{\prime}$ and $A B$ are on the neutral axis, there will no change in length; hence, Equation (4) is written as:

$$
A^{\prime} B^{\prime}=A B
$$

By substituting Equation (2) in Equation (3), Equation (5) can be written:

$$
\varepsilon_{x}=\frac{c}{R}
$$

Since the beam is only subject to moments and it is in static equilibrium, the forces across the cross-section surface are entirely longitudinal (Fig. 3). The force on each small area in the cross-sectional area is given by Equation (6):

$$
\Delta P=\sigma_{x} \cdot b \cdot d y
$$

where $\sigma_{x}$ is the longitudinal stress in $P a, b$ is the width of the beam in $m$, and $d y$ is the differential in the $y$ direction.

This result in moment is shown in Equation (7): 


$$
\Delta M=c \cdot\left(\sigma_{x} \cdot b \cdot d y\right)
$$

where $c$ is the perpendicular distance from the neutral axis in $m$.

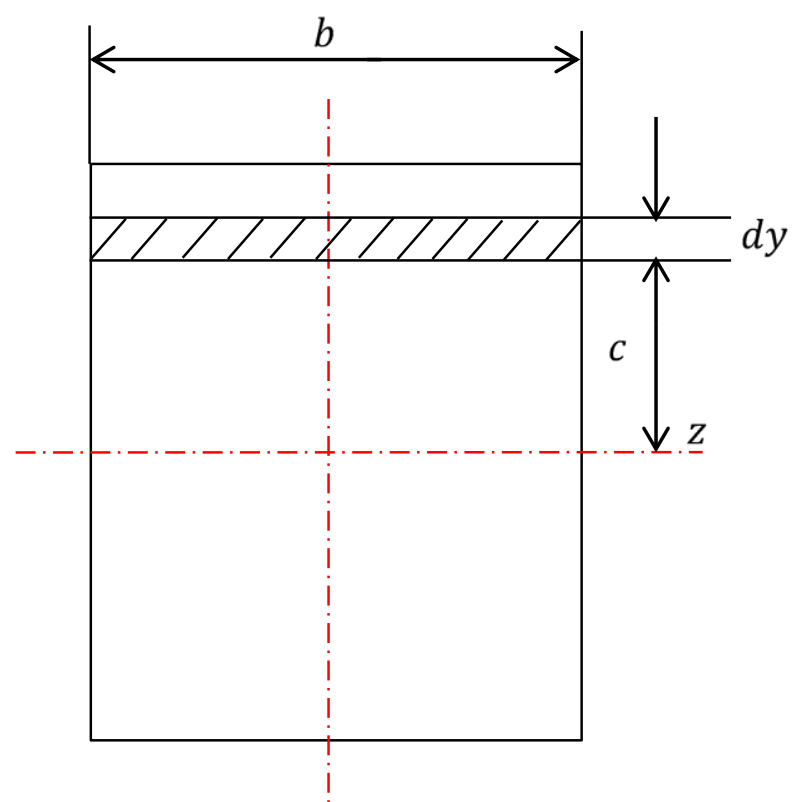

FIGURE 3. Bending moment in the cross-sectional area of a beam

By summing the moment over the complete cross-sectional area, Equation (8) is given as:

$$
M=\sum\left(\sigma_{x} \cdot c \cdot b \cdot d y\right)
$$

Considering the elasticity of the material, using Hooke's law [3], Equation (9) is given as:

$$
\sigma_{x}=E \varepsilon_{x}
$$

where $E$ is Young's modulus in $P a$.

By substituting Equation (5) in Equation (9), $\sigma_{x}$ can be re-written in Equation (10):

$$
\sigma_{x}=E \frac{c}{R}
$$

Figure 4 shows the shape of the neutral axis when the beam is bending.

As it is known, when the angle is very small, $\tan \theta=\frac{d y}{d x} \operatorname{can}$ be written as $\theta=\frac{d y}{d x}$. By the definition of $\theta$ in radians $\left(\theta=\frac{s}{R}\right.$, where $s$ is length of arc and $R$ is radius), since $d s$ is very small so $d x=d s$, resulting in Equation (11):

$$
\frac{1}{R}=\frac{d \theta}{d s}=\frac{d \theta}{d x}=\frac{d^{2} y}{d x^{2}}
$$

By substituting Equation (10) and Equation (11) into Equation (8), Equation (12) is given as: 


$$
M=\frac{E}{R} \sum c^{2} b \cdot d y=\frac{E}{R} . I
$$

where $I$ is the moment of inertia in $\left(\mathrm{m}^{4}\right)$.

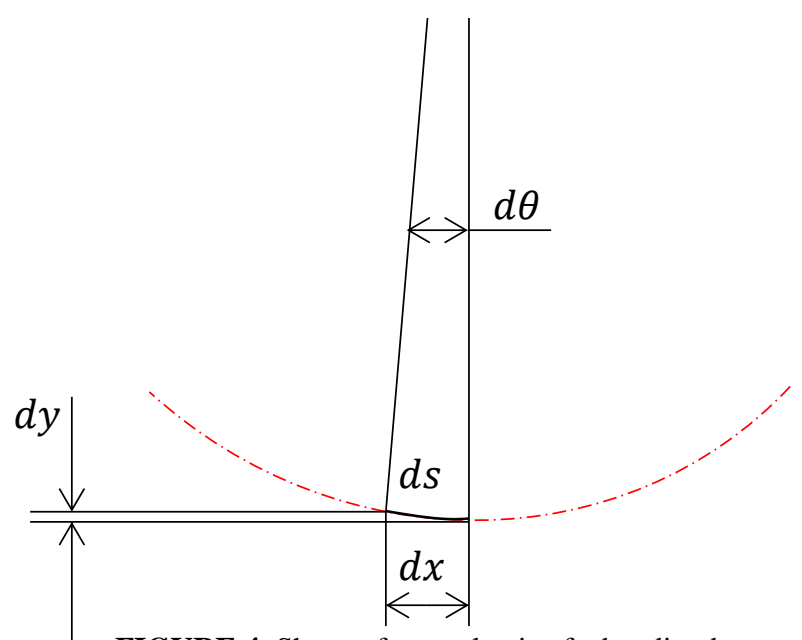

FIGURE 4. Shape of neutral axis of a bending beam

By substituting Equation (11) in Equation (12), Equation (13) is derived as shown.

$$
\frac{d^{2} y}{d x^{2}}=\frac{M}{E I}
$$

Since it is known that $\theta=\frac{d y}{d x}$, Equation (13) can be rewritten in the form of Equation (14):

$$
\theta=\int \frac{M}{E I} d x
$$

In the end, the displacement $y$ can be derived as shown in the form of Equation (15):

$$
y=\int \theta d x=\iint \frac{M}{E I} d x
$$

These equations [4] will later be applied to derive the correlation of displacement in the four-point bending beam.

In four-point bending [5, 6], a total force is applied to two locations at equal distance from the supports placed at two ends of the beam, as shown in Fig. 5. The resulted shear force and the bending moment are also shown in Fig. 5.

The advantage of four-point bending is that the moment is constant in the middle of the beam, however, it is function of $\mathrm{x}$ at both ends [7] as shown in Equation (16):

$$
\begin{gathered}
M(x)=\frac{P x}{2} \quad 0 \leq x \leq L_{1} \\
M=\frac{P L_{1}}{2} \quad L_{1} \leq x \leq\left(L-L_{1}\right) \\
M(x)=\frac{P(L-x)}{2} \quad\left(L-L_{1}\right) \leq x \leq L
\end{gathered}
$$


where $P$ is the total load in four-point bending in $N, L_{1}$ is the distance between the supporting points and the loading points on each side in $m$, and $L$ is the distance between the supports in $m$ as shown in Fig. 5 .

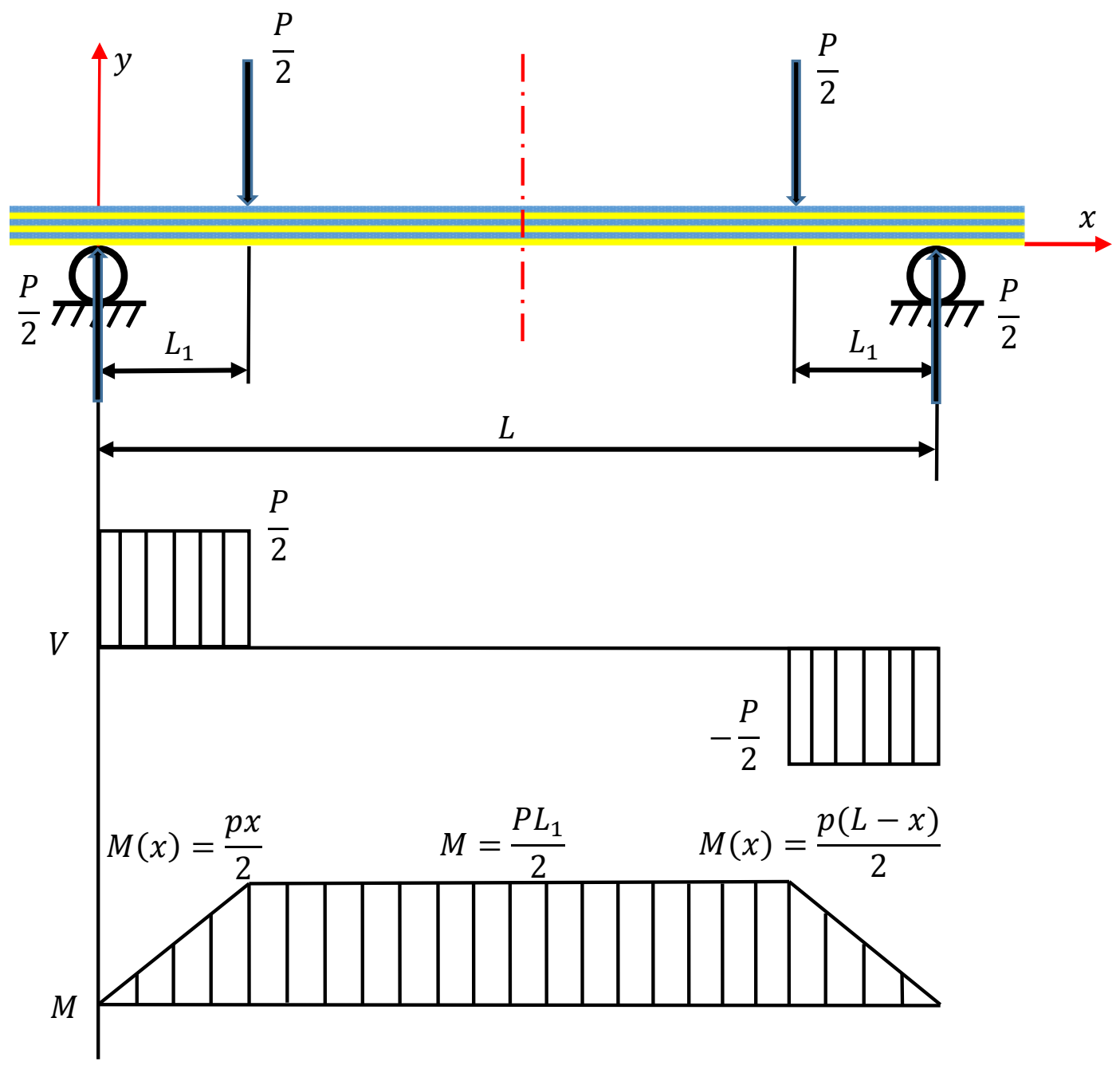

FIGURE 5. Bending moment $(M)$ and shear force $(V)$ diagrams of a four-point bending beam

By substituting Equations (16) in Equations (14) and (15), the following correlations can be derived as shown in Equations (17) to (22).

When $0 \leq x \leq L_{1}$ and $M=\frac{P x}{2}$,

$$
\begin{gathered}
\theta_{1}=\frac{P x^{2}}{4 E I}+C_{1} \\
\delta_{1}=\frac{P x^{3}}{12 E I}+C_{1} x+C_{2}
\end{gathered}
$$

When $L_{1} \leq x \leq\left(L-L_{1}\right)$ and $M=\frac{P L_{1}}{2}$,

$$
\begin{gathered}
\theta_{2}=\frac{P L_{1} x}{2 E I}+C_{3} \\
\delta_{2}=\frac{P L_{1} x^{2}}{4 E I}+C_{3} x+C_{4}
\end{gathered}
$$


When $\left(L-L_{1}\right) \leq x \leq L$ and $M=\frac{P(L-x)}{2}$,

$$
\begin{gathered}
\theta_{3}=-\frac{P x^{2}}{4 E I}+\frac{P L x}{2 E I}+C_{5} \\
\delta_{3}=-\frac{P x^{3}}{12 E I}+\frac{P L x^{2}}{4 E I}+C_{5} x+C_{6}
\end{gathered}
$$

Since we have six unknowns $C_{1}, C_{2}, C_{3}, C_{4}, C_{5}$, and $C_{6}$ we need atleast six boundary conditions (BCs) to solve the equations to get the lateral displacement $\delta$ and angular displacement $\theta$. The BCs are given in Equations (23) to (27).

$$
\begin{gathered}
x=0, \quad \delta_{1}=0 \\
x=L_{1}, \quad \delta_{1}=\delta_{2}, \quad \theta_{1}=\theta_{2} \\
x=L / 2, \quad \theta_{2}=0 \\
x=L-L_{1}, \quad \delta_{2}=\delta_{3}, \quad \theta_{2}=\theta_{3} \\
x=L, \quad \delta_{3}=0
\end{gathered}
$$

By solving the equations [8], the following results of lateral and angular displacements are obtained, as shown in Equations (28) to (33),

$$
\begin{array}{cc}
\theta_{1}=\frac{P x^{2}}{4 E I}+\frac{P L_{1}^{2}}{4 E I}-\frac{P L_{1} L}{4 E I} & \left(0 \leq x \leq L_{1}\right) \\
\delta_{1}=\frac{P x^{3}}{12 E I}+\frac{P L_{1}^{2} x}{4 E I}-\frac{P L_{1} L x}{4 E I} & \left(0 \leq x \leq L_{1}\right) \\
\theta_{2}=\frac{P L_{1} x}{2 E I}-\frac{P L L_{1}}{4 E I} & L_{1} \leq x \leq\left(L-L_{1}\right) \\
\delta_{2}=\frac{P L_{1} x^{2}}{4 E I}-\frac{P L L_{1} x}{4 E I}+\frac{P L_{1}^{3}}{12 E I} & \left(\left(L-L_{1}\right) \leq x \leq L\right) \\
\theta_{3}=-\frac{P x^{2}}{4 E I}+\frac{P L x}{2 E I}-\frac{P L_{1}^{2}}{4 E I}-\frac{P L^{2}}{4 E I}+\frac{P L L_{1}}{4 E I} & \left(\left(L-L_{1}\right) \leq x \leq L \leq L_{1} \leq L_{1}\right) \\
\delta_{3}=-\frac{P x^{3}}{12 E I}+\frac{P L x^{2}}{4 E I}-\frac{P L_{1}^{2} x}{4 E I}-\frac{P L^{2} x}{4 E I}+\frac{P L L_{1} x}{4 E I}+\frac{P L^{3}}{12 E I}+\frac{P L_{1}^{2} L}{4 E I}-\frac{P L^{2} L_{1}}{4 E I} & \left(L L^{2}\right.
\end{array}
$$

where $L$ is the distance between the supports, $P$ is the total load of four-point bending, $E$ is the Young's modulus and $I$ is the moment of inertia.

In this study, a beam is analyzed by overlaying two different materials together to form a sandwich structure. Each layer of the material is uniformly distributed throughout and perfectly bonded, free of voids. The lamina is initially in a stress-free state (no residual stresses) and behaves as linear elastic material. 


\section{ANALYTICAL STUDY}

Stress calculations in the beam are performed with respect to the neutral axis. The neutral axis of the beam goes through the centroid of its cross-section [9]. The centroid can be calculated using correlations given in Equations (34) to (36):

$$
\begin{gathered}
C_{z}=\frac{\int z d A}{A} \\
C_{y}=\frac{\int y d A}{A} \\
A=\int f(z) d z \quad y=f(z)
\end{gathered}
$$

where $C_{z}, C_{y}$ are the coordinates of the centroid; $A$ is the area; $z, y$ are the values of the z-coordinate and $y$ coordinate, respectively; $f(z)$ is a function which describes the shape. Since the beam is symmetric, $C_{z}$, the coordinate of the centroid on the $\mathrm{z}$-axis, is in the center.

In this study, the beam is made of two different materials with thicknesses $t_{1}$ and $t_{2}$ and areas $A_{1}$ and $A_{2}$, respectively, as shown in Fig. 6 (a). The number of sandwiched layers was analyzed, as shown in Fig.6 (b), (c) and (d). In these samples, the total thickness was kept constant and the individual material thicknesses were divided equally by the number of sandwiched layers, $s$; for example, $s=1$ for Fig. 6 (a), $s=2$ for Fig.6 (b) and $s=3$ for Fig. 6 (c).

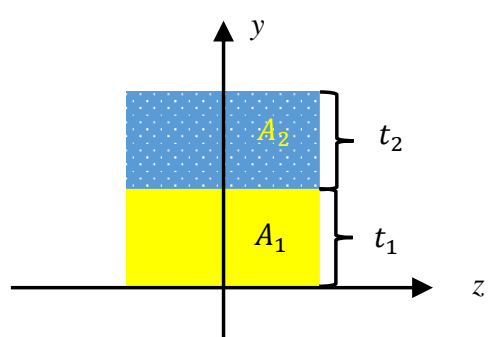

(a)

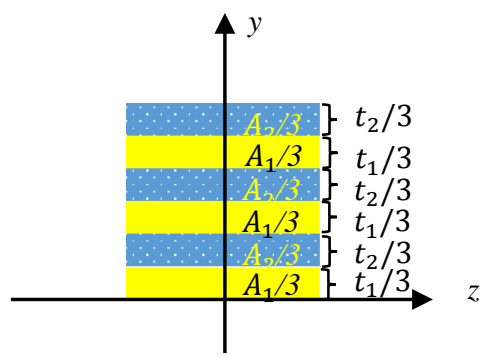

(c)

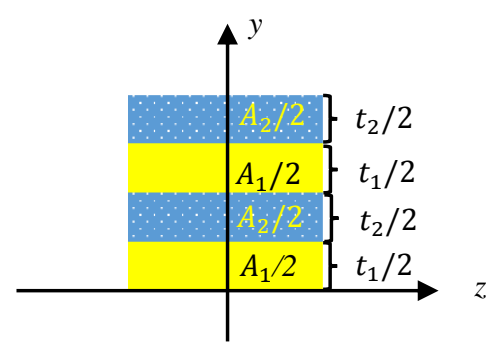

(b)

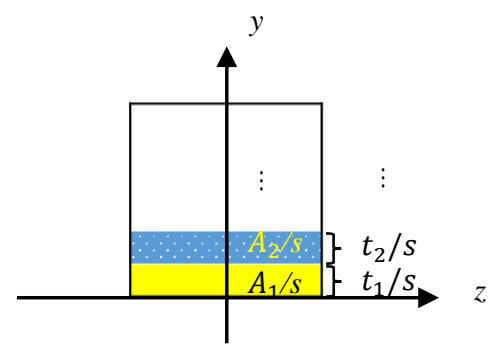

(d)

FIGURE 6. The cross-sectional area of a beam with different numbers of sandwiched layers

It is valid to assume that, under tensile loading, the Young's modulus $E$ of the beam with total cross-sectional area $A$ can be described as shown in Equation (37):

$$
E=E_{1} \frac{A_{1}}{A}+E_{2} \frac{A_{2}}{A}
$$

where $E_{1}$ and $E_{2}$ are Young's moduli of different materials with the respective net cross-sectional areas, $A_{1}$ and $A_{2}$, respectively. 
This beam contains two kinds of different materials. When it is bending, different materials have different stiffness because of the different Young's modulus, E. Therefore, the Rule of Mixtures [10] is introduced to find the combined material properties.

One of the methods of analyzing the composite beams is to use an equivalent area to represent the increase (or decrease) in stiffness. Therefore, it is important to bring in the conception of the balance coefficient, $n$ [11]. The new equivalent cross-section is assumed to be made completely from the first material, and the balance coefficient, $n$ is multiplied by the area of the second material for scaling the stiffness difference, as shown in Fig. 7.

The expansion factor, also known as the balance coefficient $n$, is given in Equation (38):

$$
n=\frac{E_{2}}{E_{1}} \quad E_{1}>E_{2} \text { (assumed) }
$$

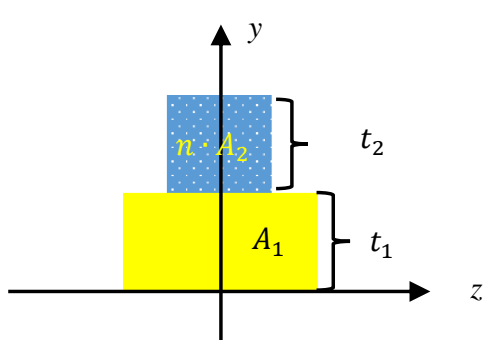

(a)

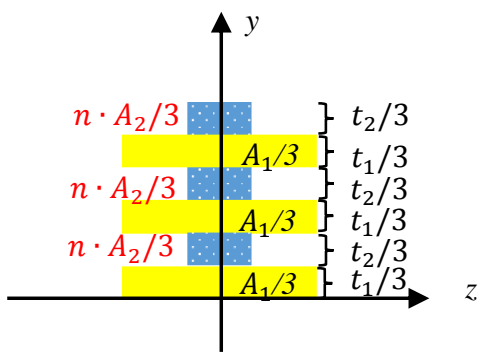

(c)

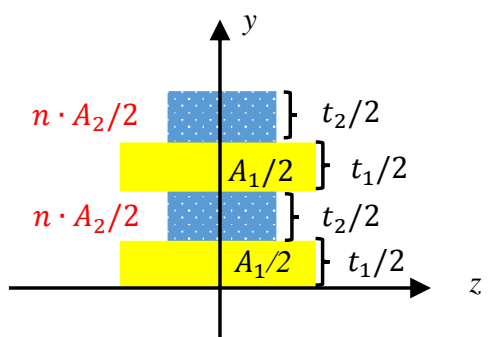

(b)

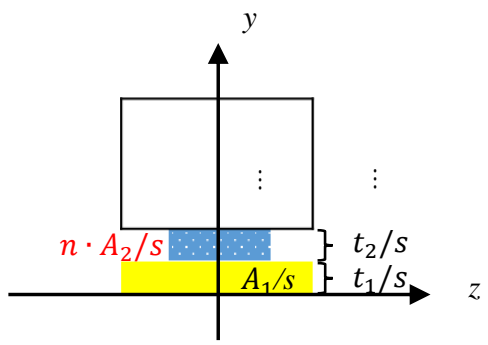

(d)

FIGURE 7. Applying balance coefficient to scale the areas

The location of the centroid and the moment of inertia change because of the difference in the Young's moduli. The new value of centroid, $C_{y}$, is calculated as shown in Equation (39):

$$
C_{y}=\frac{A_{1} \cdot \sum D_{i}+n \cdot A_{2} \cdot \sum D_{i+1}}{s \cdot\left(A_{1}+n \cdot A_{2}\right)}
$$

where $i=1,3,5,7, \ldots, 2 s-1, D_{i}$ and $D_{i+1}$ are the centroid coordinates of each layer and calculated as shown in Equations (40) to (43):

$$
\begin{gathered}
D_{1}=\frac{t_{1}}{2 s} \\
D_{2}=\frac{t_{1}}{\mathrm{~s}}+\frac{t_{2}}{2 \mathrm{~s}} \\
\vdots \\
D_{i}=D_{i-2}+\frac{t_{1}}{\mathrm{~s}}+\frac{t_{2}}{s}, \quad \text { when } s \geq 2
\end{gathered}
$$




$$
D_{i+1}=D_{i-1}+\frac{t_{1}}{\mathrm{~s}}+\frac{t_{2}}{s}, \quad \text { when } s \geq 2
$$

The moment of inertia of each layer can be calculated using the parallel axis theorem [12, 13], as shown in Equation (44):

$$
I=I_{N \cdot A}+y^{2} A
$$

where $I$ is the moment of inertia for each layer, $I_{N . A}$ is the local moment of inertia of the layer, $y$ is the distance from the neutral axis, and $A$ is the cross-sectional area of the layer.

All the moment of inertia terms can then be added together to calculate the total moment of inertia $I_{t}$ of the lamina, as shown in Equation (45):

$$
I_{t}=\sum\left(I_{1}, I_{2}, \ldots, I_{2 s-1}, I_{2 s}\right)
$$

where $I_{1}, I_{2}, \cdots, I_{2 s-1}, I_{2 s}$ are given in Equations (46) to (49):

$$
\begin{gathered}
I_{1}=\frac{b \cdot\left(\frac{t_{1}}{s}\right)^{3}}{12}+b \cdot \frac{t_{1}}{s} \cdot\left(D_{1}-C_{y}\right)^{2} \\
I_{2}=\frac{n \cdot b \cdot\left(\frac{t_{2}}{s}\right)^{3}}{12}+n \cdot b \cdot \frac{t_{2}}{s} \cdot\left(D_{2}-C_{y}\right)^{2} \\
\vdots \quad b \cdot\left(\frac{t_{1}}{s}\right)^{3} \\
I_{2 s-1}=\frac{t_{1}}{12} \cdot\left(D_{2 s-1}-C_{y}\right)^{2}, \quad \text { when } s \geq 2 \\
I_{2 s}=\frac{n \cdot b \cdot\left(\frac{t_{2}}{s}\right)^{3}}{12}+n \cdot b \cdot \frac{t_{2}}{s} \cdot\left(D_{2 s}-C_{y}\right)^{2}, \quad \text { when } s \geq 2
\end{gathered}
$$

where $t_{1}$ and $t_{2}$ are the thicknesses of material 1 and material 2,s is half of the number of layers of the beam (number of sandwiches), $b$ is the width of the material 1, $n$ is the balance coefficient, and $C_{y}$ is the position of the neutral axis of the composite beam, as given in Equation (39).

The longitudinal stresses can also be determined from the basic beam bending equation [14], as given in Equation (1). The longitudinal stresses in each layer are given in Equations (50) and (51):

$$
\begin{gathered}
\sigma_{x, 1}=\frac{M\left|y-C_{y}\right|}{I_{t}} \\
\sigma_{x, 2}=\frac{n M\left|y-C_{y}\right|}{I_{t}}
\end{gathered}
$$

where $\sigma_{x, 1}$ and $\sigma_{x, 2}$ are the longitudinal stresses in the first material and the second material, respectively, and $y$ is the position based on the reference axis (placed at the bottom of the sample). Please note that the positive value of $\left(y-C_{y}\right)$ indicates compressive longitudinal stresses and negative value of $\left(y-C_{y}\right)$ indicates tensile longitudinal stresses.

Similarly, deflection and angles can be calculated using Equations (28) to (33) by substituting the lamina's Young's modulus and moment of inertia. 
The maximum deflection is in the center at $x=\frac{L}{2}$ and can be calculated by substituting the value of $x$ in Equation (31). The maximum deflection $\delta_{\max }$ is given in Equation (52):

$$
\delta_{\text {max }}=\delta_{\text {center }}=\frac{P L_{1}}{48 E I_{t}}\left(4 L_{1}^{2}-3 L^{2}\right)
$$

where $\delta_{\text {center }}$ is the deflection in the centre, $L$ is the total length, $L_{1}$ is the distance between the support point and the loading point, $E$ is the combined Young's modulus (Equation (37)) and $I_{t}$ is the total moment of inertia about the neutral axis (Equation (45)).

\section{CONCLUSION}

In this study, analytical correlations for displacements and longitudinal stress are derived from the EulerBernoulli beam equation for a four-point bending of a sandwich structure. Appropriate initial and boundary conditions are specified to enclose the problem. The Rule of Mixtures is applied to calculate the position of the neutral axis and the moment of inertia for a sandwich structure. The resulting correlation can be used to calculate the displacements and longitudinal stress at any point in a complex sandwich beam.

\section{ACKNOWLEDGMENT}

The author would like to acknowledge the support of Linda March from The Good English Company, UK for proofreading this work.

\section{REFERENCES}

[1] S. Park, X. Gao. Bernoulli-Euler beam model based on a modified couple stress theory. Journal of Micromechanics and Microengineering. 2006;16(11):2355.

[2] O. A. Bauchau, J. I. Craig. Structural Analysis: With Applications to Aerospace Structures: Springer Netherlands; 2009.

[3] T. M. Atanackovic, A. Guran. Theory of Elasticity for Scientists and Engineers: Birkhäuser Boston; 2012.

[4] H. Xue, H. Khawaja, M. Moatamedi. Multiphysics design optimization for aerospace applications: Case study on helicopter loading hanger. AIP Conference Proceedings. 2014;1637(1):1211-7.

[5] J. C. Pais, J. Harvey. Four point bending: CRC Press; 2012.

[6] H. Xue, H. Khawaja. Investigation of Ice-PVC separation under Flexural Loading using Multiphysics Analysis. The International Journal of Multiphysics. 2016;10(3):In Press.

[7] J. B. Wachtman, W. R. Cannon, M. J. Matthewson. Mechanical properties of ceramics: John Wiley \& Sons; 2009.

[8] R. Budynas, W. Young, A. Sadegh. Roark's Formulas for Stress and Strain, 8th Edition: McGraw-Hill Education; 2011.

[9] C. Hartsuijker, J. W. Welleman. Engineering Mechanics: Volume 2: Stresses, Strains, Displacements: Springer Netherlands; 2007.

[10] W. Soboyejo. Mechanical properties of engineered materials: CRC press; 2002.

[11] R. C. Hibbeler, K. S. V. Sekar. Mechanics of Materials: Pearson Education Canada; 2013.

[12] R. M. Jones. Mechanics of composite materials: Scripta Book Company Washington, DC; 1975.

[13] O. W. Eshbach, B. D. Tapley, T. R. Poston. Eshbach's handbook of engineering fundamentals: John Wiley \& Sons; 1990.

[14] D. M. Mohan. Basic Engineering Mechanics and Strength of Materials: PHI Learning Pvt. Ltd.; 2010. 